\title{
Imaging and non-imaging Cherenkov hybrid reconstruction
}

\author{
Douglas R Bergman ${ }^{* a}$ and Tareq Abu-Zayyad ${ }^{a}$ for the Telescope Array \\ Collaboration ${ }^{\dagger}$ \\ ${ }^{a}$ The University of Utah \\ E-mail: bergman@physics.utah.edu \\ E-mail: tareq@eosmic.utah.edu
}

\begin{abstract}
Air showers with primary energies between 3 and $100 \mathrm{PeV}$ which are pointed toward TALE give rise to an optical signal dominated by Cherenkov radiation rather than fluorescence light. The reconstruction of these showers can be greatly improved for a sample of these showers by placing a small (400 m square) array of non-imaging Cherenkov counters ( 25 counters) below the field of view of TALE. Estimates of the hybrid reconstruction resolutions for shower geometry, energy and $X_{\max }$ are presented along with estimates of the hybrid aperture. NICHE counter designs and construction plans will be presented.
\end{abstract}

The 34th International Cosmic Ray Conference,

30 July- 6 August, 2015

The Hague, The Netherlands

\footnotetext{
${ }^{*}$ Speaker.

${ }^{\dagger}$ Full author list at: http://www.telescopearray.org/images/papers/ICRC2015-authorlist.pdf
} 


\section{Imaging Cherenkov for CRs from 1-100 PeV}

Imaging Air Cherenkov Telescopes (IACTs) have been used to observe the Extensive Air Showers (EASs) produced by TeV gamma rays for some time[1]. In these measurements the much more common Cosmic Ray (CR) produced EASs are a background. Typical IACTs are steerable with a field-of-view of a few degrees and are used in arrays so that the EASs can be observed in stereo. For these observations, it is important that the cameras in the IACTs have a small pixel size of order a $0.1^{\circ}$ in order to differentiate between photon produced EASs and the much more copious CR produced showers.

At PeV energies, the flux of photons is expected to be very small, but IACTs can still be used to observe CR EASs. Because of the lower fluxes at high energies, having a large field-of-view becomes of primary importance in obtaining a significant number of events. This comes at the expense however, of being able to make stereo observations.

As it happens the Telescope Array (TA) Low Energy extension (TALE) has telescopes designed for observing sub-EeV CRs in fluorescence. These telescopes are also ideal IACTs for measuring PeV CRs[2]. Unlike the TeV IACTs, TALE observes the Cherenkov light from high $P_{T}$ electrons in the shower, i.e., observes the shower from well outside the Cherenkov cone defined by the core of the shower. The ideal viewing angles are $1.5^{\circ}-5^{\circ}$. This has the effect of making the observed tracks much longer than for TeV IACTs, which is ideally suited to the much large pixels, approximately $1^{\circ}$, of TALE.

The difficulty in reconstructing showers with TALE is that in monocular measurements, one needs very long observed tracks in order to accurately determine the shower geometry within the shower-detector plane. However, when looking almost directly back along the shower as TALE does in Cherenkov measurements, a small change in the geometry makes a very large change in the depth of the imaged point of the shower. This can make the measured profile completely unphysical. Using this fact, and the fact that EAS longitudinal profiles from hadronic cosmic rays are all very similar, with a narrow range of depths of maxima and a small range of widths, one can use the profile as a constraint to allow a quite accurate measurement of the EAS geometry. This is called the Profile Constrained Geometry Fit (PCGF). However, one would like to have confirmation that the PCGF is working properly, and when it is, of the uncertainty in the fit EAS properties that the PCGF imposes.

\section{Non-imaging Cherenkov Observations}

In addition to making an image of the EAS in Cherenkov light, one can sample the Cherenkov light at many points on the ground. The index-of-refraction of the atmosphere is such that Cherenkov cones from the core of the EAS and from heights above the ground from 8-20 km all form rings of $120-140 \mathrm{~m}$ on the ground. The actual $P_{T}$ of the shower electrons smears out this ring, but the $\sim 120 \mathrm{~m}$ mark still separates the inner part of the Cherenkov shower footprint, filled in from the late, low part of the shower below $8 \mathrm{~km}$, from the outer part of the shower which has contributions from higher- $P_{T}$ electrons throughout the EAS. The ratio of inner to outer Cherenkov intensities or the Cherenkov Lateral Distribution (CLD) thus is connected to details of the EAS longitudinal profile. Unlike surface detector arrays (which observe the actual electrons of showers) 
the Cherenkov photons in the outer region come from all parts of the shower, not just the last few radiation lengths of the atmosphere.

The time structure of the Cherenkov signal at a given point in the outer part of the shower also depends on the longitudinal profile. The portion of the shower which gives a measurable signal in a given counter depends on where the shower is brightest. If this is lower in the atmosphere, the time span in which the photons arrive will be longer.

To use non-imaging Cherenkov, one typically uses an array of collectors. To measure the inner part of the shower footprint well, these collectors should have a spacing less than $120 \mathrm{~m}$. However, larger spacings may still be useful if one intends to use the Cherenkov Time Width Distribution (CTWD) to measure the properties of air showers. At PeV energies, there is significant Cherenkov flux at the ground that the collectors can be of order $10-\mathrm{cm}$ in size.

The Non-Imaging CHErenkov (NICHE) array is a proposed array to make just this sort of measurement[3]. However, using TALE and NICHE together will be even better.

\section{Benefits of Cherenkov hybrid}

The imaging and non-imaging air Cherenkov measurement techniques can be used together very effectively in the PeV region. This is similar to the way hybrid surface detector and fluorescence detector measurements work together at EeV scales, but the synergies are somewhat different, since both parts of the Cherenkov hybrid obtain signals from the bulk of the EAS.

Like the EeV-scale hybrid detectors, PeV Cherenkov hybrid has excellent geometric reconstruction accuracy due to the addition of multiple timing measurements on the ground. This avoids the necessity of using the PCGF in TALE. In addition, because both the imaging and non-imaging detectors will make independent energy and $X_{\max }$ measurements of the EAS, one can constrain other uncertainties such as the angular distribution of Cherenkov light within the EAS (c.f. the poster by Bergman from the 33rd ICRC[4]).

\section{Single prototype counter measurements}

With TALE now deployed and commissioned, we constructed a prototype Cherenkov counter using two detectors from the BLANCA experiment. It should be pointed out that the 3.5" PMTs used in these are from the Fly's Eye detector, thus over 30 years old, and may have a significantly reduced sensitivity.

In the NICHE-BLANCA prototype, the two BLANCA counters were placed side-by-side on a shipping pallet, and then tilted up to an angle of $45^{\circ}$ to match the median TALE viewing angle. Note that the BLANCA counters have a viewing angle of $12.5^{\circ}$ rather than the $45^{\circ}$ planned for the actual NICHE array. The larger entrance aperture of the Winston cones, however, compensates for the low sensitivity of the PMTs. The PMT signals from both BLANCA counters were fed into a CAEN 12-bit digitizer operating at 250 MSPS. A coincidence between the two signals was required for triggering. An external $1 \mathrm{~Hz}$ trigger was provided by GPS for synchronization to TALE. Power was provided by a portable generator. A picture of the NICHE-BLANCA prototype in the field is shown in Figure 1. 


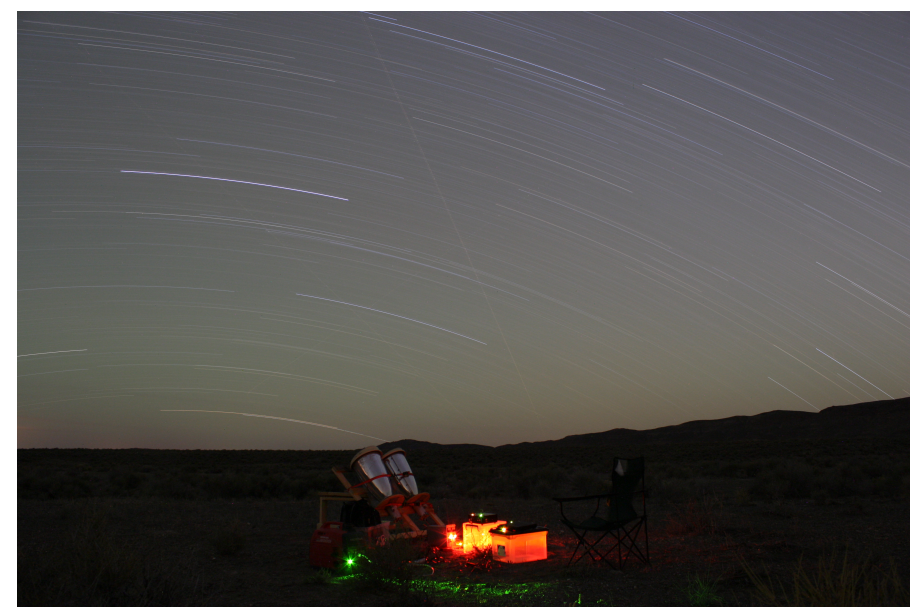

Figure 1: The NICHE-BLANCA Prototype in the field. The two BLANCA counters are evident from their long Winston cones, strapped to a shipping pallet aligned to point up at $45^{\circ}$. The green light is from the portable generator used for power, while the red lights are from HV power supplies.

On the morning of 17 February 2015, we placed the NICHE-BLANCA prototype approximately $15 \mathrm{~m}$ in front of TALE and pointed in the direction as two of the TALE mirrors in order to look for coincidences between the two detectors. At the close position, the coincident events are not track-like in TALE, so the normal TALE analysis could not be performed. However, most of the signal in TALE comes within a few hundred ns (TALE's sample period is $100 \mathrm{~ns}$ ), so a simple sum of the signal sufficed for performing a timing coincidence. A typical coincident event is shown on the left in Figure 2. Because the TALE signal is likely to be strongest and earliest in the center, with later signals filling out the circular collection of PMTs contributing, we compare the NICHE-BLANCA prototype trigger time to the mode of the TALE times. The resulting distribution of times is shown on the right in Figure 2. The RMS of 55 ns compares well with a perfect match (given the $100 \mathrm{~ns}$ TALE sample period) of an RMS of $29 \mathrm{~ns}$. We will fold the 29-ns offset (different from the 29-ns perfect RMS) into the expected time in the far-position analysis below. The offset includes PMT transit time and trigger latency from both sites. The 103 coincident events were obtained in approximately 10 hours of running.

On the morning of 18 February 2015 we placed the NICHE-BLANCA prototype approximately $680 \mathrm{~m}$ from TALE, again pointed in the same direction as the TALE mirrors. The coincidences found in this location are track-like in TALE, so we used the full TALE (PCGF) analysis. It should be noted that the PCGF analysis provides a very tight constraint on the angle of these events $\left(\sim 0.1^{\circ}\right)$ but the impact parameter distance is less constrained and susceptible to uncertainty from "bad tubes" (PMTs with trigger times determined from night-sky background or other noise sources). A typical coincident event is shown in three panels in Figure 3. The left panel shows the result of the PCGF fit of TALE with the NICHE-BLANCA time projected to the TALE position. The 29-ns offset from near-position measurements is included the 55-ns RMS is shown as the error. This point is not included in the fit, but we will look to see how different it is from the fit projection. The center panel shows the geometry of the event as projected onto the ground plane of TALE. The shower core is represented as an arrow with the point of the arrow being where the 

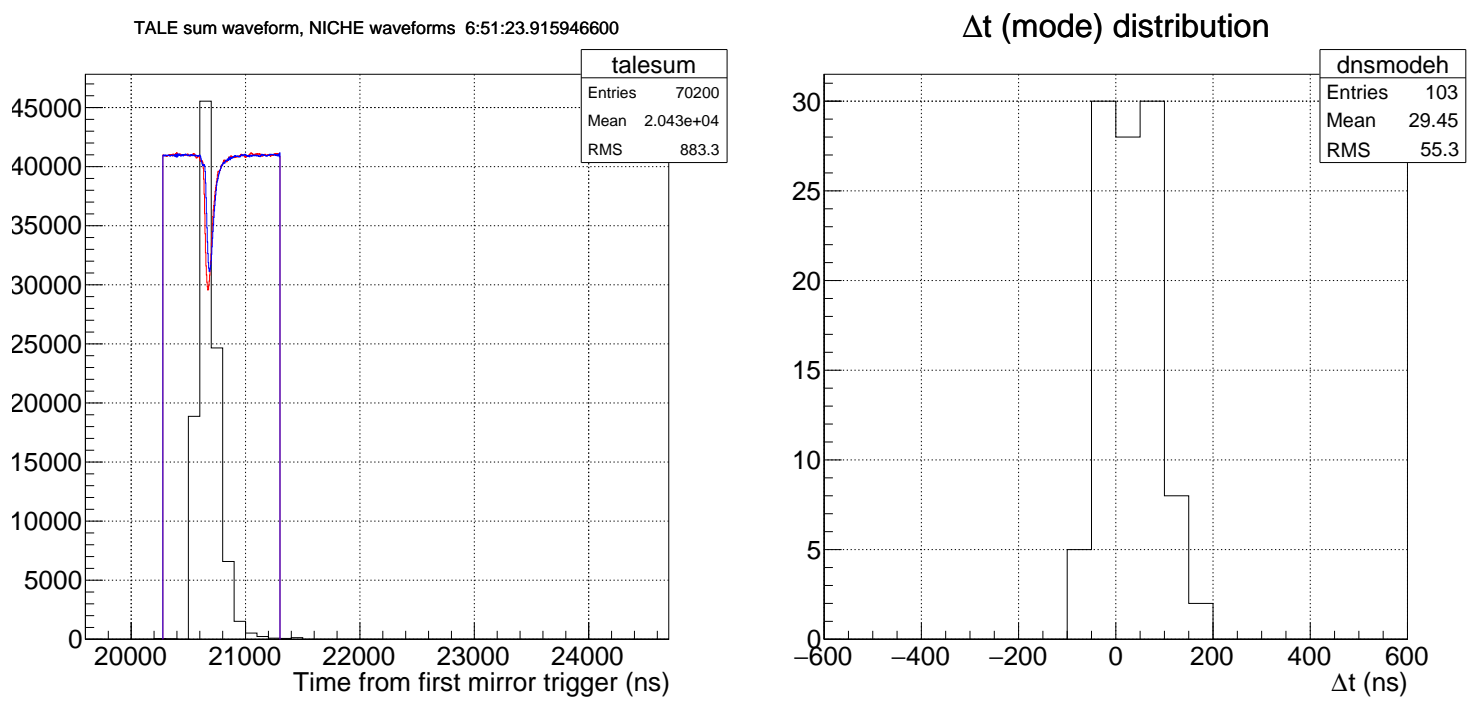

Figure 2: Left: A coincidence between TALE and the NICHE-BLANCA prototype in the close position. The TALE signal is the sum of all PMT-signal FADC counts in triggered mirrors. The NICHE-BLANCA waveform is then overlaid in red and blue (one for each counter). The mean baseline signal from the NICHEBLANCA FADC is scaled to be $90 \%$ of the TALE maximum. Right: The difference in time between the NICHE-BLANCA trigger and the mode of the TALE (summed) signal.

shower hits the ground. The length of the arrow is $200 \mathrm{~m} \mathrm{long}$, but projected onto the ground plane to give some sense of the zenith angle of the shower. The horizontal positions of TALE and the NICHE-BLANCA prototype are shown along with the points on the shower axis closest to each (as projected onto the ground plane). The right panel shows the view from TALE. The PMTs contributing are shown by colored circles, with the size of the circle proportional to the signal and the color (blue to red) indicating the time. The line shows the projection of the shower-detector plane onto the alt-azimuth plane. The NICHE-BLANCA prototype position is also indicated.

We were fortunate to observe one very high energy event, observed in fluorescence from TALE. This event is shown in Figure 4. Note that the shower is not pointed into the NICHEBLANCA prototype at all, but scattered Cherenkov light and the lateral extent of the shower were enough to trigger the counter.

We observed 17 coincidences in approximately 10 hours at the far position. A summary of these events is shown in Figure 5. The left panel shows the difference between the NICHEBLANCA time and the prediction for what it should have been given the PCGF fit at TALE. The 288-ns RMS is larger than one would like to see, but this is not yet a hybrid geometry fit. With only one counter on the ground, we cannot constrain the core position very well, so no hybridgeometry fit was performed. The center panel shows the locations of the where the showers hit the ground. For the most part they fall all around the NICHE-BLANCA prototype as expected. The one outlier in the SE is effected by a "bad tube" and should likely be close. The right panel shows the distribution of PCGF-reconstructed energies. The threshold appears to be about $3 \mathrm{PeV}$. The exact contribution to the threshold of TALE and the NICHE-BLANCA prototype has yet to be determined. The one event above $100 \mathrm{PeV}$ is the fluorescence event shown in Figure 4. 

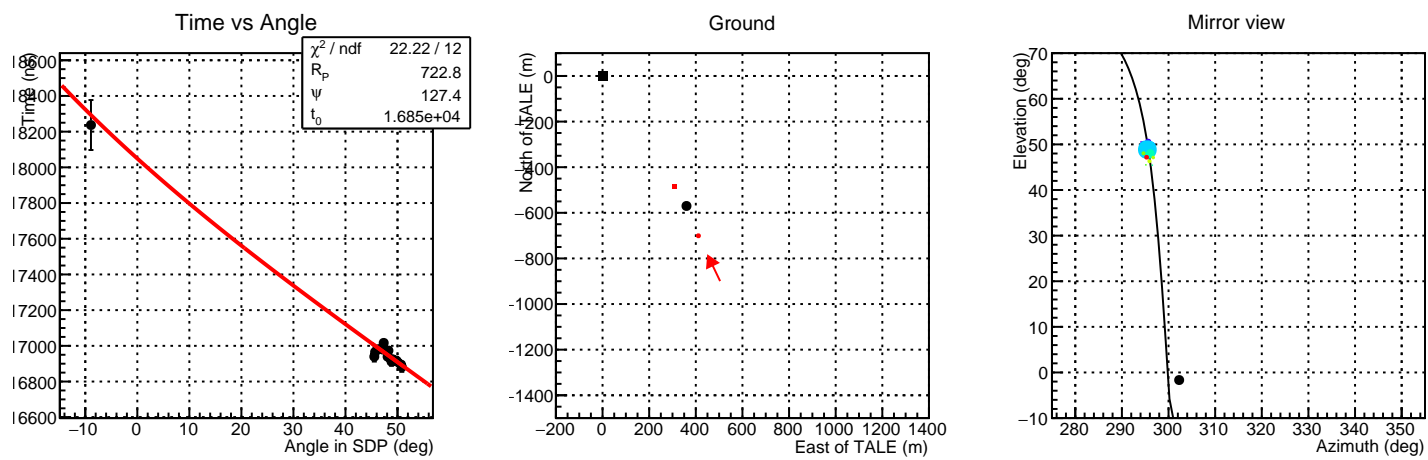

Figure 3: A typical Cherenkov hybrid event. Left: Hybrid time-versus-angle. The time of light reaching TALE versus the angle in the shower-detector plane at which the light came. The BLANCA-NICHE prototype time and angle are adjusted for the projection of the NICHE counter onto the shower track. The red line shows the TALE-only profile-constrained fit (with fit parameters shown). Middle: Projection onto the TALE horizontal plane of the TALE position (black square), the BLANCA-NICHE prototype position (black circle), and the shower impact point and shower direction (red arrow, point of arrow at impact point). The location of the point where the shower axis is closest to TALE (the impact parameter point) as projected to the surface is shown as a small red square. The point where the shower axis passes closes to BLANCANICHE is shown as a small red circle. Note that since all the red elements are projections from the shower axis, they are all colinear in the figure. Right: The view on the sky. The pointing direction of PMTs viewing the shower, with the size being proportional to the received signal and the color going with time. The shower-detector plane is shown as the black line.
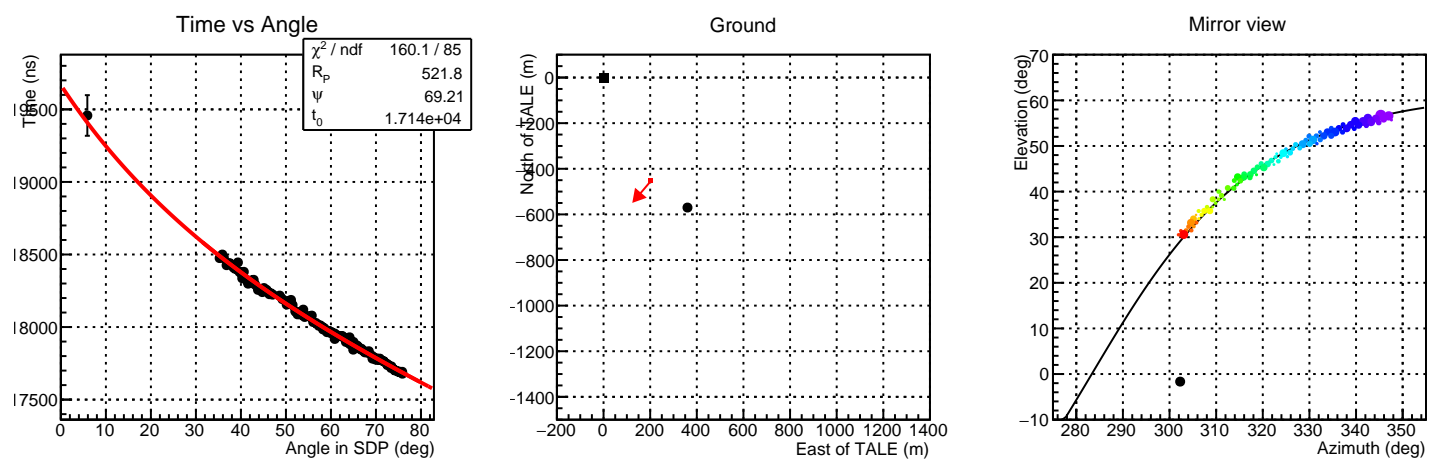

Figure 4: A fluorescence event. The panels are as in Figure 3. Note that the shower is not pointing at either TALE or BLANCA-NICHE.

\section{Conclusion}

Both imaging and non-imaging Cherenkov detection methods are suitable for observing CR EASs at PeV energies. By using both imaging and non-imaging Cherenkov detectors in a hybrid detector, and even stronger observation can be made. We have deployed and commissioned the TALE detector as an IACT, and we are working to obtain funding for NICHE, a non-imaging Cherenkov array to be placed in front of TALE and work with it in hybrid.

To assess the feasibility of NICHE counters, we have built a prototype NICHE counter using 

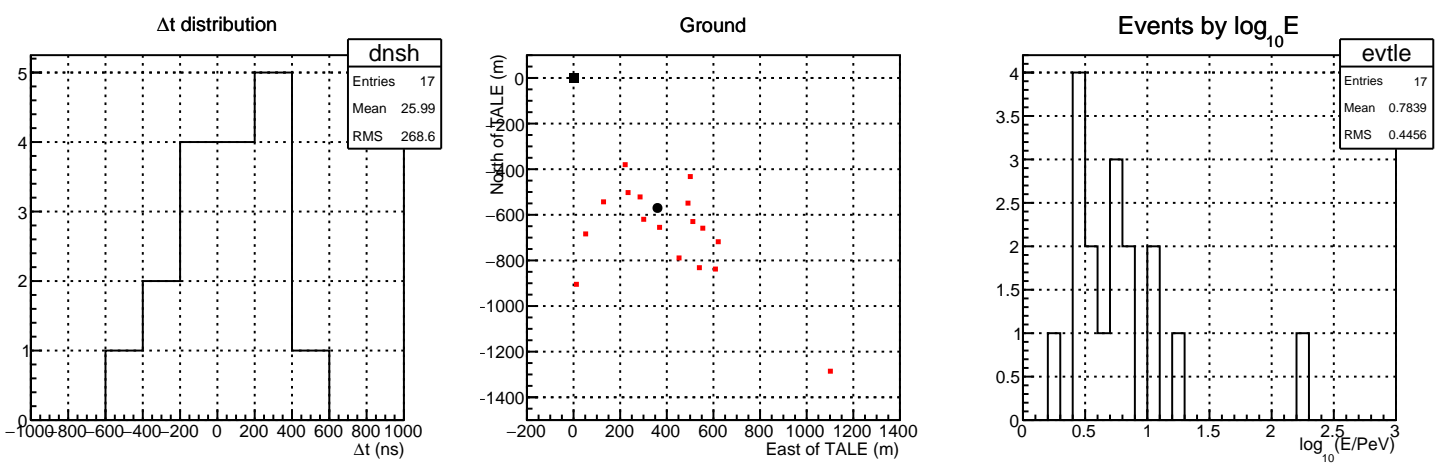

Figure 5: Summary plots of the 17 events seen in coincidence by TALE CHerenkov and NICHE-BLANCA Left: The difference between the NICHE-BLANCA time and the prediction for what it should have been given the PCGF fit at TALE Middle: Locations of shower cores in the TALE horizontal plane (red squares). TALE is shown as a black square and NICHE-BLANCA is shown as a black circle. Right: The energies of the events as estimated from TALE.

old BLANCA counters. We succeeded in finding coincidences between this NICHE-BLANCA prototype and TALE.

\section{Acknowledgements}

The Telescope Array experiment is supported by the Japan Society for the Promotion of Science through Grants-in-Aid for Scientific Research on Specially Promoted Research (21000002) "Extreme Phenomena in the Universe Explored by Highest Energy Cosmic Rays" and for Scientific Research (19104006), and the Inter-University Research Program of the Institute for Cosmic Ray Research; by the U.S. National Science Foundation awards PHY-0307098, PHY-0601915, PHY-0649681, PHY-0703893, PHY-0758342, PHY-0848320, PHY-1069280, PHY-1069286, PHY1404495 and PHY-1404502; by the National Research Foundation of Korea (2007-0093860, R3210130, 2012R1A1A2008381, 2013004883); by the Russian Academy of Sciences, RFBR grants 11-02-01528a and 13-02-01311a (INR), IISN project No. 4.4502.13, and Belgian Science Policy under IUAP VII/37 (ULB). The foundations of Dr. Ezekiel R. and Edna Wattis Dumke, Willard L. Eccles, and George S. and Dolores Doré Eccles all helped with generous donations. The State of Utah supported the project through its Economic Development Board, and the University of Utah through the Office of the Vice President for Research. The experimental site became available through the cooperation of the Utah School and Institutional Trust Lands Administration (SITLA), U.S. Bureau of Land Management, and the U.S. Air Force. We also wish to thank the people and the officials of Millard County, Utah for their steadfast and warm support. We gratefully acknowledge the contributions from the technical staffs of our home institutions. An allocation of computer time from the Center for High Performance Computing at the University of Utah is gratefully acknowledged. 


\section{References}

[1] J. Holder, Ground-Based Gamma Ray Astronomy, Proceedings of the 33rd ICRC (Rio de Janeiro), Brazilian Journal of Physics 44 (2014) 450.

[2] T. Abu-Zayyad, Cerenkov Events Seen by The TALE Air Fluorescence Detector, Proceedings of DPF 2013 (Santa Cruz), arXiv:1310.0069.

[3] D.R. Bergman, J.F. Krizmanic, Y. Tsunesada, The NICHE Array: status and plans, these proceedings, PoS(ICRC2015)635.

[4] D.R. Bergman, An Efficient Technique for the Reconstruction of Extensive Air Showers using Non-Imaging Cherenkov Measurements, Proceedings of the 33rd ICRC (Rio de Janeiro), 0983. 